DOE/ER/12119--2

DE 93012237

\title{
SYNTHESIS OF NEW HIGH PERFORMANCE LUBRICANTS AND SOLID LUBRICANTS
}

\author{
Progress Report
}

Apri1 1992 - March 1993

Professor Richard J. Lagow

Department of Chemistry

The University of Texas at Austin

Austin, Texas 78712-1167

\section{Apri1 1993}

\section{PREPARED FOR THE U.S. DEPARTMENT OF ENERGY} UNDER GRANT NUMBER DE-FG05-91ER12119

\section{DISCLAIMER}




\section{SYNTHESIS OF NEH HIGH PERFORMANCE LUBRICANTS AND SOLID LUBRICANTS}

\section{Technical Progress Report}

In our second year of funding we began the testing phase of a number of new classes of lubricants. Three different testing collaborations have already begun and a fourth one is in the works with Dr. Stephen Hsu of the National Institute of Standards and Technology with whom we had established a working relationship after meeting at the Automotive and Technology Development Coordination Meeting held in November 2-5, 1992 in Dearborn, Michigan. Dr. Hsu also plans to test some of the same materials for us that Shell Development is studying.

With Dr. Bill Jones of NASA, we are studying the effects of branching on high temperature lubricant properties in perfluoropolyethers. Initially Bill Jones is comparing the Tubrication and physical properties of perfluorotetraglyme and the following two spherical perfluoropolyethers. Note that one contains a fluorocarbon chain and the other one contains a fluorocarbon ether chain. The synthesis of these was reported in the last progress report.
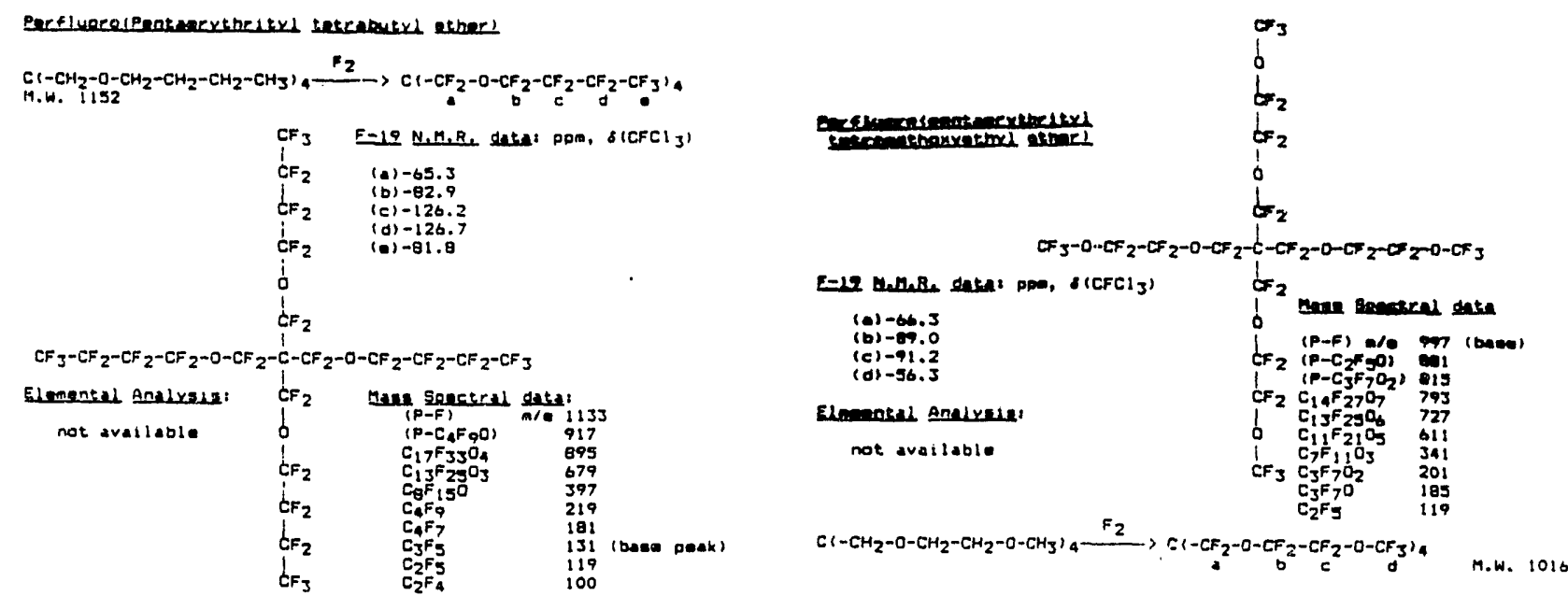
With Professor Patricia Thiel of Iowa State Universicy, we are working on studies of perfluoromethylene oxide ethers and have prepared a series of four of these polyethers to study in collaboration with her research group. These are model compounds which correspond to structures proposed on the bottom of page 17 and top of page 18 of our research proposal:

$$
\begin{aligned}
& \left(-C F_{2} O-C F_{2} C F_{2} C F_{2}-O-\right)_{n} \\
& \left(-C F_{2} O-C F_{2} C F_{2} C F_{2} C F_{2}-0-\right)_{n}
\end{aligned}
$$<smiles>CC(F)(F)C(C)(C)C(=O)O</smiles><smiles>[3H][C]([13CH3])O[14CH3]</smiles>

These perfluoromethylene oxide ethers have the best low temperature properties of any known lubricants. Thiel's group is studying their interactions with metals under extreme conditions.

Thirdly, we have also begun an interaction with Dr. August Birke of She11 Development Company in Houston for whom we have already prepared samples of the chlorine-substituted fluorocarbon polyether lubricants whose structures appear on page 54 of our research proposal. Each of these four structures is thought to have potential as lubricant additives to motor oils. Each of the following structures is completely soluble in hydrocarbon motor oils and hydrocarbon polyalphaolefins.

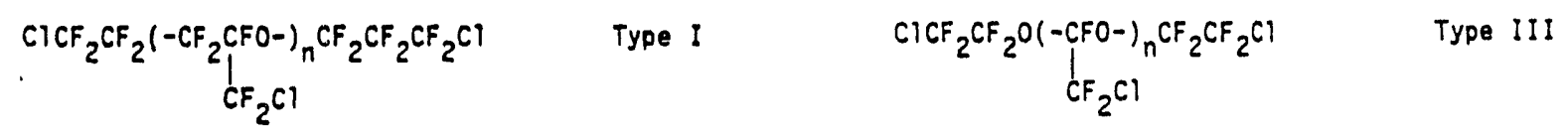

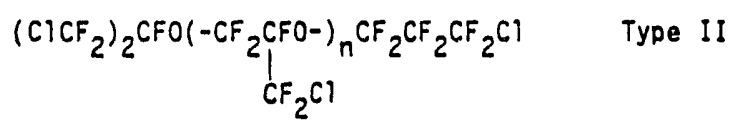

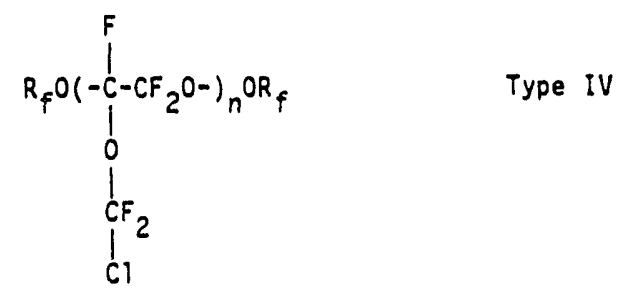


We also have underway syntheses of other fluorine-containing branched ether lubricants. These new materials which are also promising as antifriction additives for motor oils appear ahead of the perfluoro additives as Appendix I to the progress report. Additionally for Birke and Shell Development we have at their request prepared the novel compound perfluoro salicylic acid. This synthesis was suggested by the Shell staff who thought that esters of perfluoro salicylic acid might be an excellent antifriction additive for motor oil fuels. One of the best additives currently used in motor oils is the hydrocarbon ester of salicylic acid.

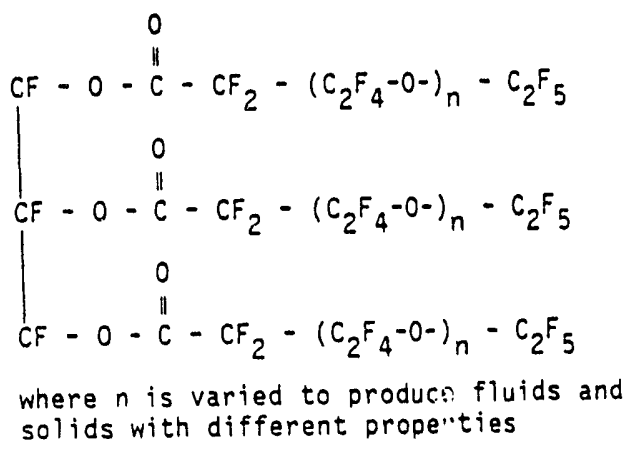

Dr. Kuangsen Sung of our research group has succeeded in preparing the first example of glycerin-based perfluoropolyester structures and specifically has succeeded in preparing the glyceride ester of perfluoro stearic acid, $\mathrm{CF}_{3}\left(\mathrm{CF}_{2}\right)_{16} \mathrm{COOH}$. We shall be submitting this new class of branched perfluorocarbon esters for testing shortly.

We also have achieved success with synthes is of perfluoro epoxy ether chains, a class of compounds that have never been previously prepared: 
<smiles>C(CCOCC1CO1)COCC1CO1</smiles><smiles>C(COCC1CO1)OCC1CO1</smiles><smiles>C1OC1C1CO1</smiles><smiles>CC(C)(COCC1CO1)COCC1CO1</smiles><smiles>C(CCC1CO1)CC1CO1</smiles>

Additionally with Dr. Bill Jones of NASA we are testing a new class of antifriction additives for perfluoropolyether lubricants, the perfluorophosphoranes. We have made quite a number of these and will shortly be submitted these to $\mathrm{Dr}$. Jones for screening. The first structure appears below and the rest of the new structures constitute Appendix II.

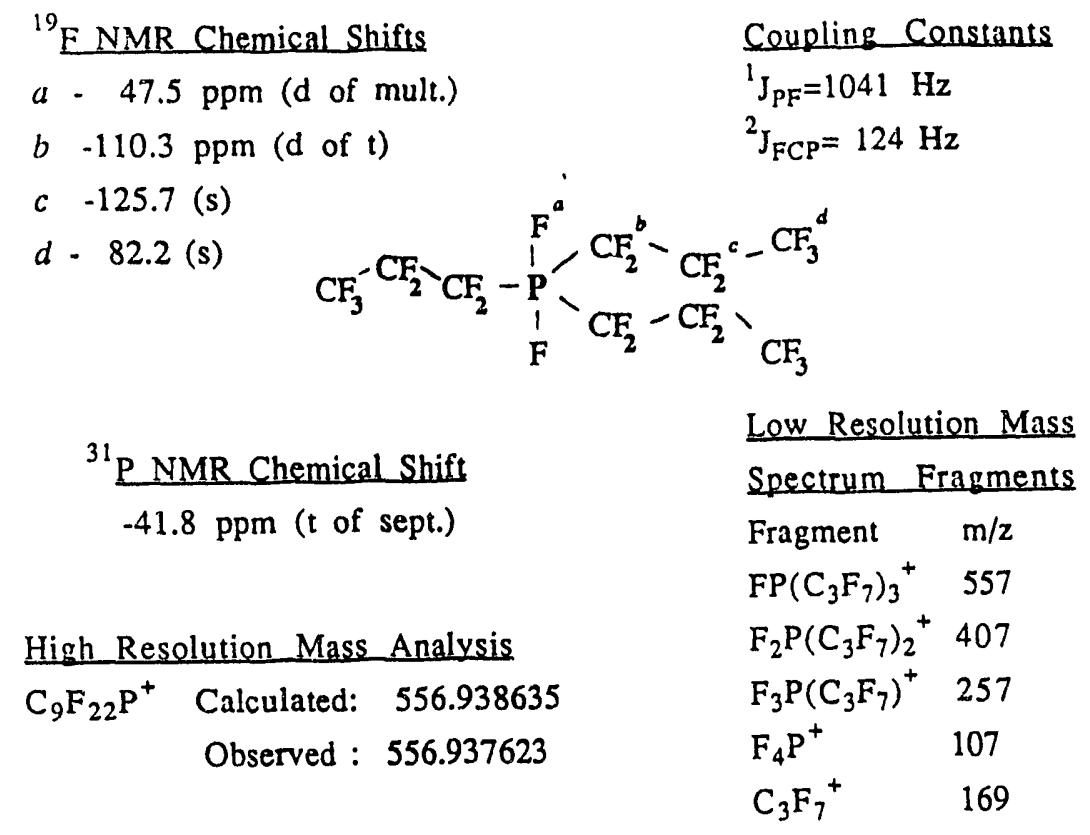

There are successes in many other areas to report but we lack the space here to do so. This has been a very successful year in our program. 
APPENDIX I

General Scheme

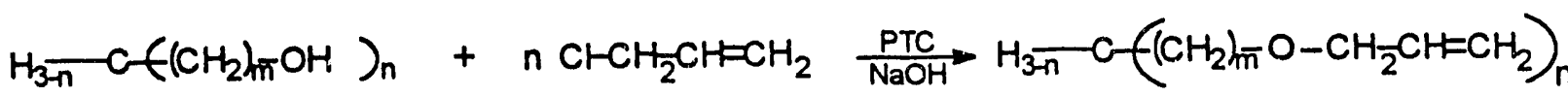

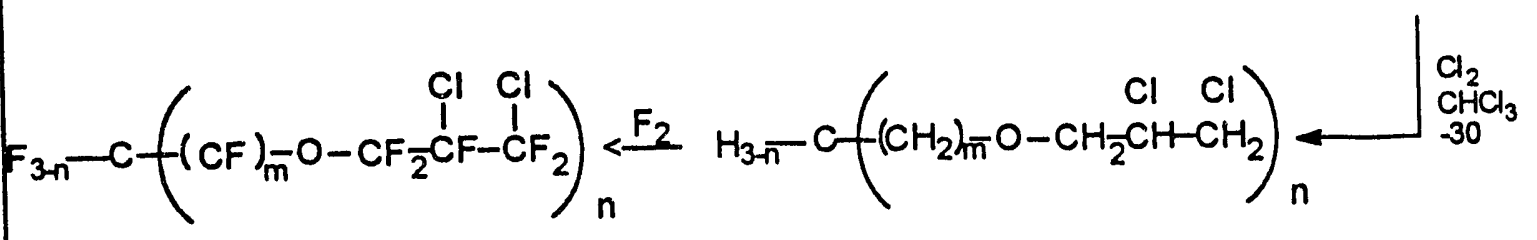


Representative examples:

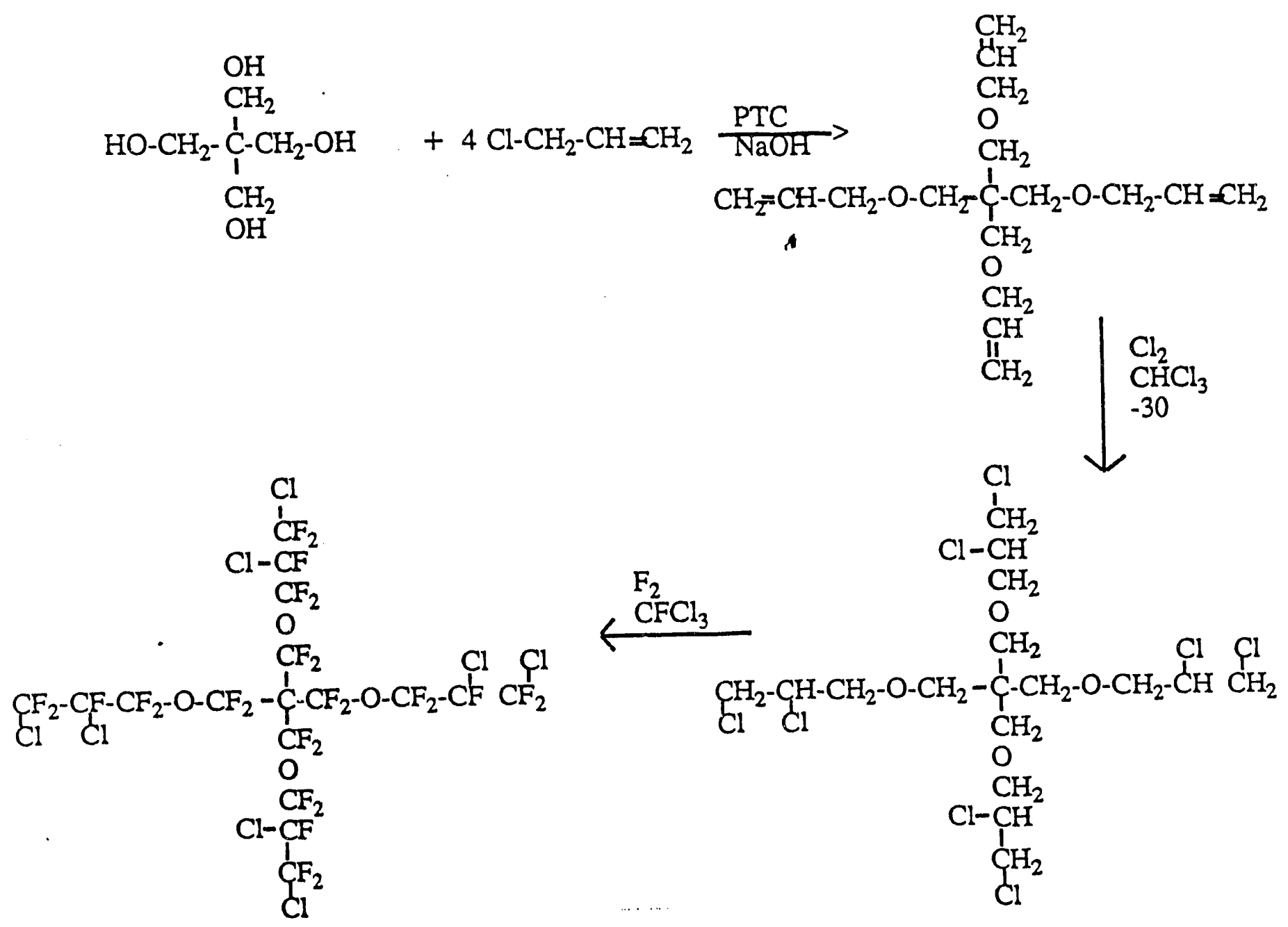

C.I. Mass Spec. Parent - F m/e $=1065$

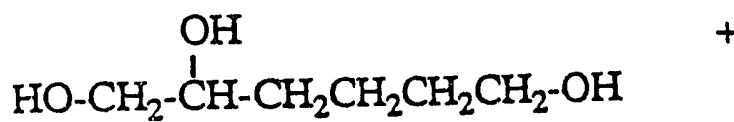

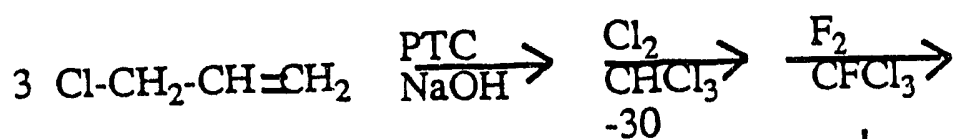
$\underset{\mathrm{Cl}-\mathrm{CF}_{2}^{\mathrm{Cl}}}{\stackrel{\mathrm{CF}}{\mathrm{CF}_{2}}}$

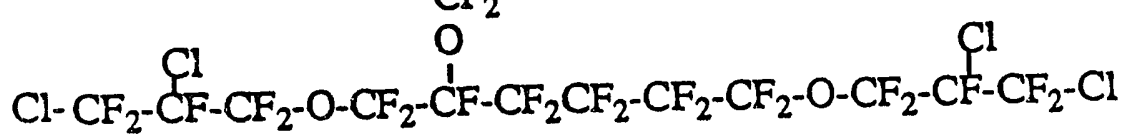

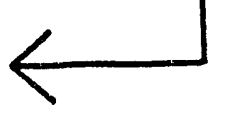

C.I. Mass. Spec. Parent $-F \mathrm{~m} / \mathrm{e}=916$ 
Variations in the polyhydrolic starting materials will provide a variety of chlorofluorocarbon ether structures. Synthesis of the following compounds is underway.

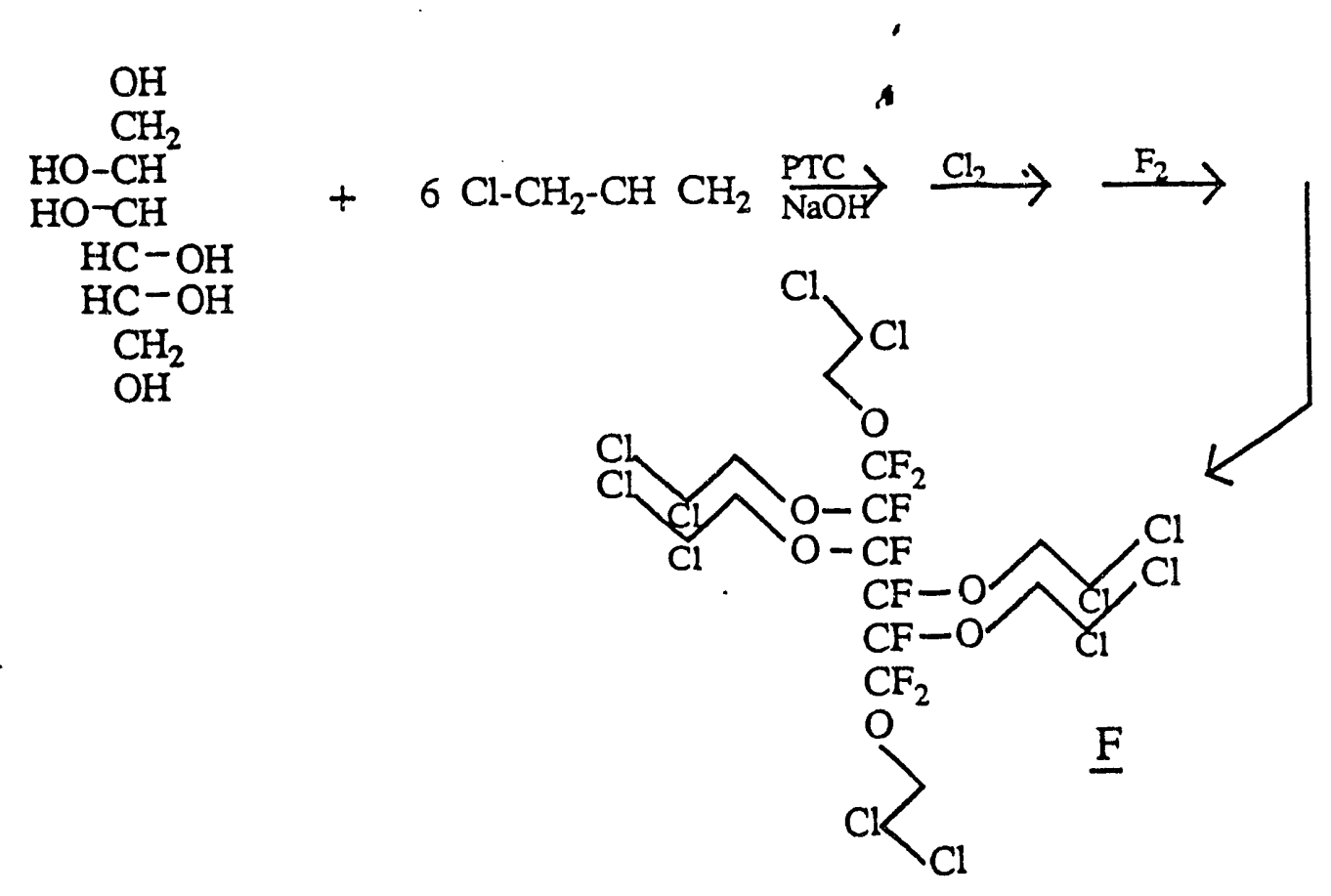

结

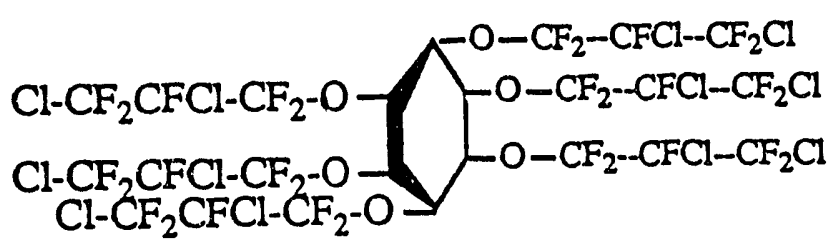

$\underline{F}$ 
Approach II : Conversion of Perfluoroesters to Perfluoroethers using $\mathrm{SF}_{4}$ is well known. Synthesis of chlorine containing fluoroesters followed by their conversion to ethers will provide for a variety of chlorinated fluoroethers, and will also provide a great deal of control over chlorine content and location. The synthesis of such molecules is underway.

$$
\text { General Scheme }
$$

$\mathrm{H}_{3-\mathrm{n}}-\mathrm{C}-(\mathrm{R}-\stackrel{\mathrm{O}}{\mathrm{C}}--\mathrm{OH})_{-\mathrm{n}}+\mathrm{n} \quad \mathrm{HO}--\mathrm{CH}_{2}-\mathrm{R}-\mathrm{CH}_{2}-\mathrm{Cl} \stackrel{\mathrm{H}^{+}}{-\mathrm{H}_{2} \mathrm{O}}$

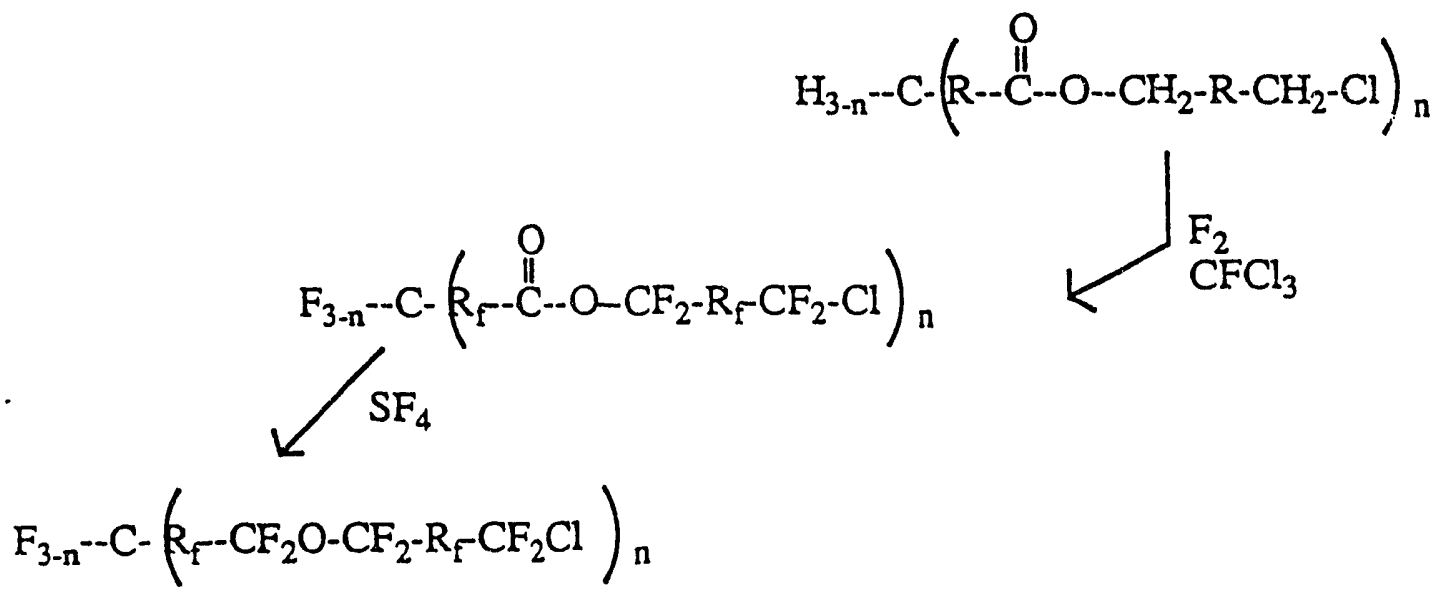

Representative examples:

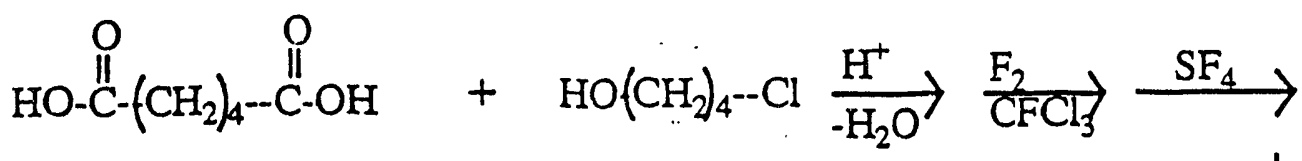

$\mathrm{Cl}-\mathrm{CF}_{2} \mathrm{CF}_{2} \mathrm{CF}_{2} \mathrm{CF}_{2}-\mathrm{O}-\mathrm{CF}_{2} \mathrm{CF}_{2} \mathrm{CF}_{2} \mathrm{CF}_{2}-\mathrm{O}-\mathrm{CF}_{2} \mathrm{CF}_{2} \mathrm{CF}_{2} \mathrm{CF}_{2}-\mathrm{Cl}$ 


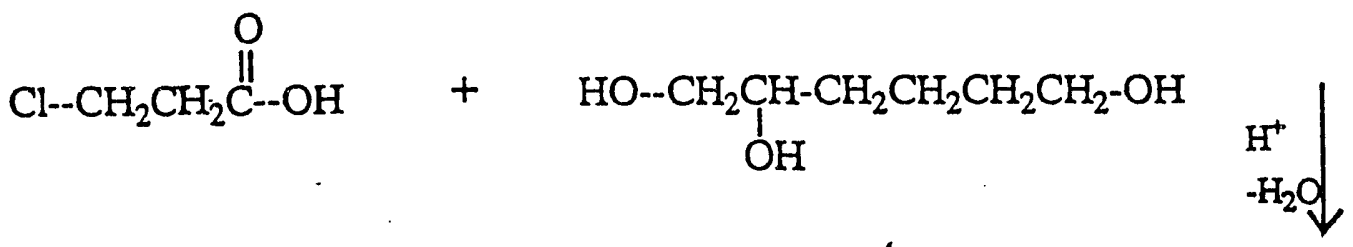

$$
\begin{aligned}
& \mathrm{Cl}_{2} \quad . \mathrm{SF}_{4} \leftarrow \frac{\mathrm{F}_{2}}{\mathrm{CFCl}_{3}} \\
& \mathrm{CF}_{2} \\
& \mathrm{CF}_{2} \\
& \mathrm{Cl}-\mathrm{CF}_{2} \mathrm{CF}_{2} \mathrm{CF}_{2}-\mathrm{O}-\mathrm{CF}_{2}-\mathrm{CF}-\mathrm{CF}_{2} \mathrm{CF}_{2} \mathrm{CF}_{2} \mathrm{CF}_{2}-\mathrm{O}-\mathrm{CF}_{2} \mathrm{CF}_{2} \mathrm{CF}_{2}-\mathrm{Cl}
\end{aligned}
$$




\section{APPENDIX II}

$$
\begin{aligned}
& { }^{19} \text { F NMR Chemical Shifts Coupling Constants } \\
& \text { a }-47.5 \mathrm{ppm} \text { (d of mult.) } \quad{ }^{1} \mathrm{~J}_{\mathrm{PF}}=1041 \mathrm{~Hz} \\
& \text { b } \quad-110.3 \mathrm{ppm} \text { (d of } \mathrm{t} \text { ) } \quad{ }^{2} \mathrm{~J}_{\mathrm{FCP}}=124 \mathrm{~Hz} \\
& \text { c }-125.7(\mathrm{~s}) \\
& \text { d }-82.2(\mathrm{~s})
\end{aligned}
$$

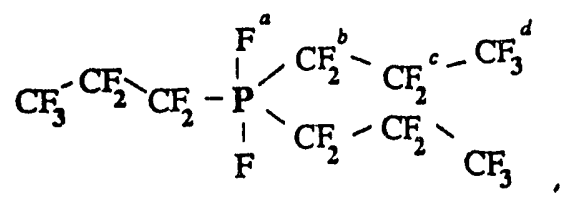

\section{${ }^{31}$ P NMR Chemical Shift $-41.8 \mathrm{ppm}$ ( $\mathrm{t}$ of sept.)}

${ }^{19}$ E NMR Chemical Shifts

$a$ - 46.8 ppm (d of mult.)

b $\quad-108.8 \mathrm{ppm}$ (d)

Coupling Constants

${ }^{1} \mathrm{~J}_{\mathrm{PF}}=1050 \mathrm{~Hz}$

${ }^{2} \mathrm{~J}_{\mathrm{FCP}}=126 \mathrm{~Hz}$

c -120.7 (s)

$d-123.4(\mathrm{~s})$

e $-127.4(s)$

$f-82.5(\mathrm{~s})$

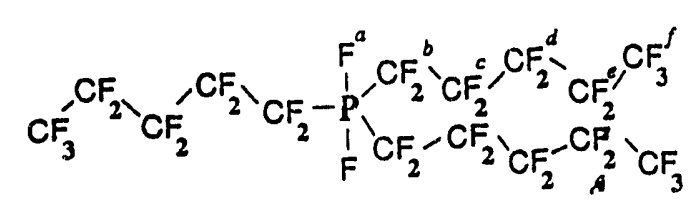

${ }^{31}$ P NMR Chemical Shift

$-37.6 \mathrm{ppm}$ ( $\mathrm{t}$ of sept.)

High Resolution Mass Analysis

$\mathrm{C}_{15} \mathrm{~F}_{34} \mathrm{P}^{+} \quad$ Calculated: 856.919474

Observed : 856.918693
Low Resolution Mass Spectrum Fragments

Fragment $\mathrm{m} / \mathrm{z}$

$\mathrm{FP}\left(\mathrm{C}_{5} \mathrm{~F}_{11}\right)_{3}{ }^{+} \quad 857$

$\mathrm{F}_{2} \mathrm{P}\left(\mathrm{C}_{5} \mathrm{~F}_{11}\right)_{2}{ }^{+} 607$

$\mathrm{F}_{3} \mathrm{P}\left(\mathrm{C}_{5} \mathrm{~F}_{11}\right)^{+} \quad 357$

$\mathrm{F}_{4} \mathrm{P}^{+} \quad 107$

$\mathrm{C}_{5} \mathrm{~F}_{11}{ }^{+} \quad 269$ 


$$
\begin{aligned}
& { }^{19} \text { F NMR Chemical Shifts } \\
& \text { a - } 46.9 \text { ppm (d of mult.) } \\
& \text { Coupling Constants. } \\
& \begin{array}{lll}
b & -109.1 \mathrm{ppm} \text { (d) }
\end{array} \\
& { }^{1} \mathrm{~J}_{\mathrm{PF}}=1060 \mathrm{~Hz} \\
& { }^{2} \mathrm{~J}_{\mathrm{FCP}}=123 \mathrm{~Hz} \\
& \text { c }-120.8(\mathrm{~s}) \\
& \text { d }-122.8(\mathrm{~s}) \\
& \text { e }-123.1(\mathrm{~s}) \\
& f-123.4(\mathrm{~s}) \\
& g \quad-124.3(s) \\
& \text { h }-128.0 \text { (s) } \\
& i \cdot 83.3 \text { (s) } \\
& { }^{31} \text { P NMR Chemical Shift } \\
& -38.0 \mathrm{ppm} \text { ( } t \text { of sept.) } \\
& \text { Low Resolution Mass } \\
& \text { Spectrum Fragments } \\
& \text { Fragment } \quad m / z \\
& \mathrm{~F}_{2} \mathrm{P}\left(\mathrm{C}_{8} \mathrm{~F}_{17}\right)_{3}^{\circ} 1326 \\
& \mathrm{FP}\left(\mathrm{C}_{8} \mathrm{~F}_{17}\right)_{3}{ }^{+} 1307 \\
& \mathrm{~F}_{2} \mathrm{P}\left(\mathrm{C}_{8} \mathrm{~F}_{17}\right)_{2}{ }^{+} 907 \\
& \mathrm{~F}_{3} \mathrm{P}\left(\mathrm{C}_{8} \mathrm{~F}_{17}\right)^{+} \quad 507 \\
& \mathrm{~F}_{4} \mathrm{P}^{+} \quad 107 \\
& \mathrm{C}_{8} \mathrm{~F}_{17}{ }^{+} \quad 419 \\
& \text { c } \quad-81.5(\mathrm{~s}) \\
& \text { d } \quad-117.2(\mathrm{~d}) \\
& e-p \quad-121.9(\mathrm{~s}) \\
& q \quad-123.0(\mathrm{~s}) \\
& r \quad-126.6(\mathrm{~s}) \\
& s \quad-81.7(s)
\end{aligned}
$$

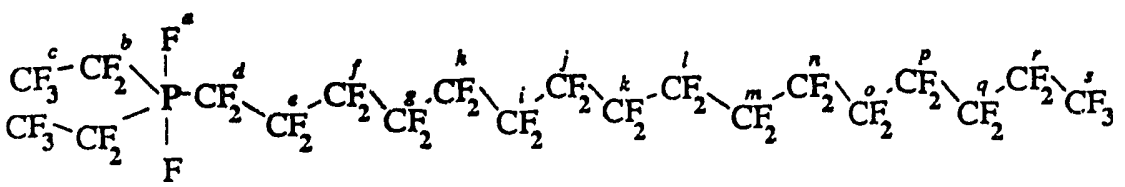

$$
\begin{aligned}
& { }^{31} \mathrm{P} \text { NMR Chemical Shift } \\
& -43.8 \mathrm{ppm} \text { ( } \mathrm{t} \text { of sept.) }
\end{aligned}
$$

\section{Spectrum Fragments}




$$
\begin{aligned}
& { }^{19} \text { E NMR Chemical Shifts Coupling Constants } \\
& \text { a }-18.7 \mathrm{ppm} \text { (d of mult.) } \quad{ }^{1} \mathrm{~J}_{\mathrm{PF}}=1015 \mathrm{~Hz} \\
& \text { b }-176.7 \mathrm{ppm} \text { (d) } \quad{ }^{2} \mathrm{~J}_{\mathrm{FCP}}=99 \mathrm{~Hz} \\
& \text { c }-71.0(\mathrm{~s})
\end{aligned}
$$

$\begin{array}{lc}\text { Fragment } & \mathrm{m} / \mathrm{z} \\ \mathrm{FP}\left(\mathrm{C}_{3} \mathrm{~F}_{7}\right)_{3}{ }^{+} & 557 \\ \mathrm{~F}_{2} \mathrm{P}\left(\mathrm{C}_{3} \mathrm{~F}_{7}\right)_{2}{ }^{+} & 407 \\ \mathrm{~F}_{3} \mathrm{P}\left(\mathrm{C}_{3} \mathrm{~F}_{7}\right)^{+} & 257 \\ \mathrm{~F}_{4} \mathrm{P}^{+} & 107 \\ \mathrm{C}_{3} \mathrm{~F}_{7}{ }^{+} & 169\end{array}$

${ }^{19}$ E NMR Chemical Shifts Coupling Constants

a - 50.2 ppm (d of mult.)

${ }^{1} \mathrm{~J}_{\mathrm{PF}}=1007 \mathrm{~Hz}$

b $\quad-114.7$ ppm (d)

${ }^{2} \mathrm{~J}_{\mathrm{FCP}}=122 \mathrm{~Hz}$

c -83.1 (s)

d -114.1 (d)

${ }^{2} \mathrm{~J}_{\mathrm{FCP}}=112 \mathrm{~Hz}$

$e-84.9(\mathrm{~s})$

$f-90.0(t)$

$g-88.9(\mathrm{~s})$

${ }^{4} \mathrm{~J}_{\mathrm{FCOCF}}=9 \mathrm{~Hz}$

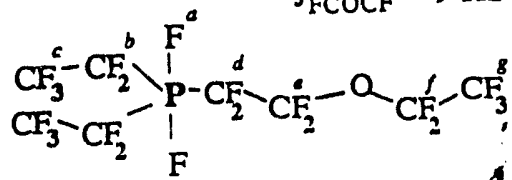

Low Resolutiôn Mass

${ }^{31}$ P N.MR Chemical Shift

Spectrum Fragments

$-45.3 \mathrm{ppm}$ ( $t$ of sept.)

Fragment

$\mathrm{F}_{2} \mathrm{P}\left(\mathrm{C}_{2} \mathrm{~F}_{5}\right)_{2}\left(\mathrm{C}_{4} \mathrm{~F}_{9} \mathrm{O}\right)^{-5} 542$

$\mathrm{FP}\left(\mathrm{C}_{2} \mathrm{~F}_{5}\right)_{2}\left(\mathrm{C}_{4} \mathrm{~F}_{9} \mathrm{O}\right)^{+} 523$

High Resolution Mass Analysis

$\mathrm{F}_{2} \mathrm{P}\left(\mathrm{C}_{2} \mathrm{~F}_{5}\right)\left(\mathrm{C}_{4} \mathrm{~F}_{9} \mathrm{O}\right)^{+} 423$

$\mathrm{C}_{8} \mathrm{~F}_{20} \mathrm{OP}^{+}$Calculated: 522.936743

${ }_{2} \mathrm{P}\left(\mathrm{C}_{2} \mathrm{~F}_{5}\right)_{2}{ }^{+} \quad 307$

Observed : 522.935822

$\mathrm{F}_{3} \mathrm{P}\left(\mathrm{C}_{2} \mathrm{~F}_{5}\right)^{+}$

$\mathrm{F}_{3} \mathrm{P}\left(\mathrm{C}_{4} \mathrm{~F}_{9} \mathrm{O}\right)^{+}$

323

$\mathrm{F}_{4} \mathrm{P}^{+}$

107

$\mathrm{C}_{4} \mathrm{~F}_{9} \mathrm{O}^{+}$

235

$\mathrm{C}_{2} \mathrm{~F}_{5}{ }^{+}$

119 


$$
\begin{array}{ll}
{ }^{19} \mathrm{~F} \text { NMR Chemical Shifts } & \frac{\text { Coupling Constants }}{2 \mathrm{~J}_{\mathrm{FCP}}=122 \mathrm{~Hz}} \\
a-83.6 \mathrm{ppm}(\mathrm{s}) & { }^{1} \mathrm{~J}_{\mathrm{PF}}=1019 \mathrm{~Hz} \\
b-114.9 \mathrm{ppm}(\mathrm{d}) & { }^{2} \mathrm{~J}_{\mathrm{FCP}}=125 \mathrm{~Hz} \\
c-49.8(\mathrm{~d} \text { of mult.) } & \\
d-110.0(\mathrm{~d}) & \\
e-120.6(\mathrm{~s}) & \\
f-128.2(\mathrm{~s}) & \\
\mathrm{CF}_{3}^{a}-\mathrm{CF}_{2}^{b} \mathrm{~F}_{\mathrm{P}}-\mathrm{CF}_{2}^{d}-\mathrm{CF}_{2}^{e}-\mathrm{CF}_{2}^{d} \mathrm{CF}_{2}-\mathrm{CF}_{2}-\mathrm{P}_{\mathrm{P}}^{-} \mathrm{CF}_{2}-\mathrm{CF}_{3} \\
\mathrm{CF}_{3}-\mathrm{CF}_{2}-\mathrm{CF}_{3}
\end{array}
$$

\section{${ }^{31}$ P NMR Chemical Shift High Resolution Mass Analysis \\ -44.0 ppm ( $t$ of sept.) $\quad \mathrm{C}_{13} \mathrm{~F}_{33} \mathrm{P}_{2}^{+} \quad$ Calculated: 844.894834 \\ Observed : 844.894530}
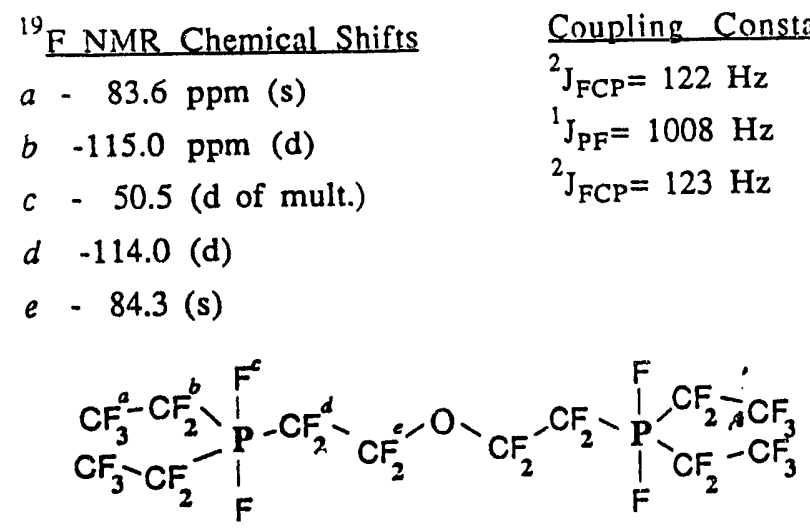

${ }^{31} \mathrm{P}$ NMR Chemical Shift $-45.2 \mathrm{ppm}$ ( $\mathrm{t}$ of sept.)
High Resolution Mass Analysis

$\mathrm{C}_{12} \mathrm{~F}_{32} \mathrm{OP}_{2}^{-}$Calculated: 829.891345

Observed : 829.893040 


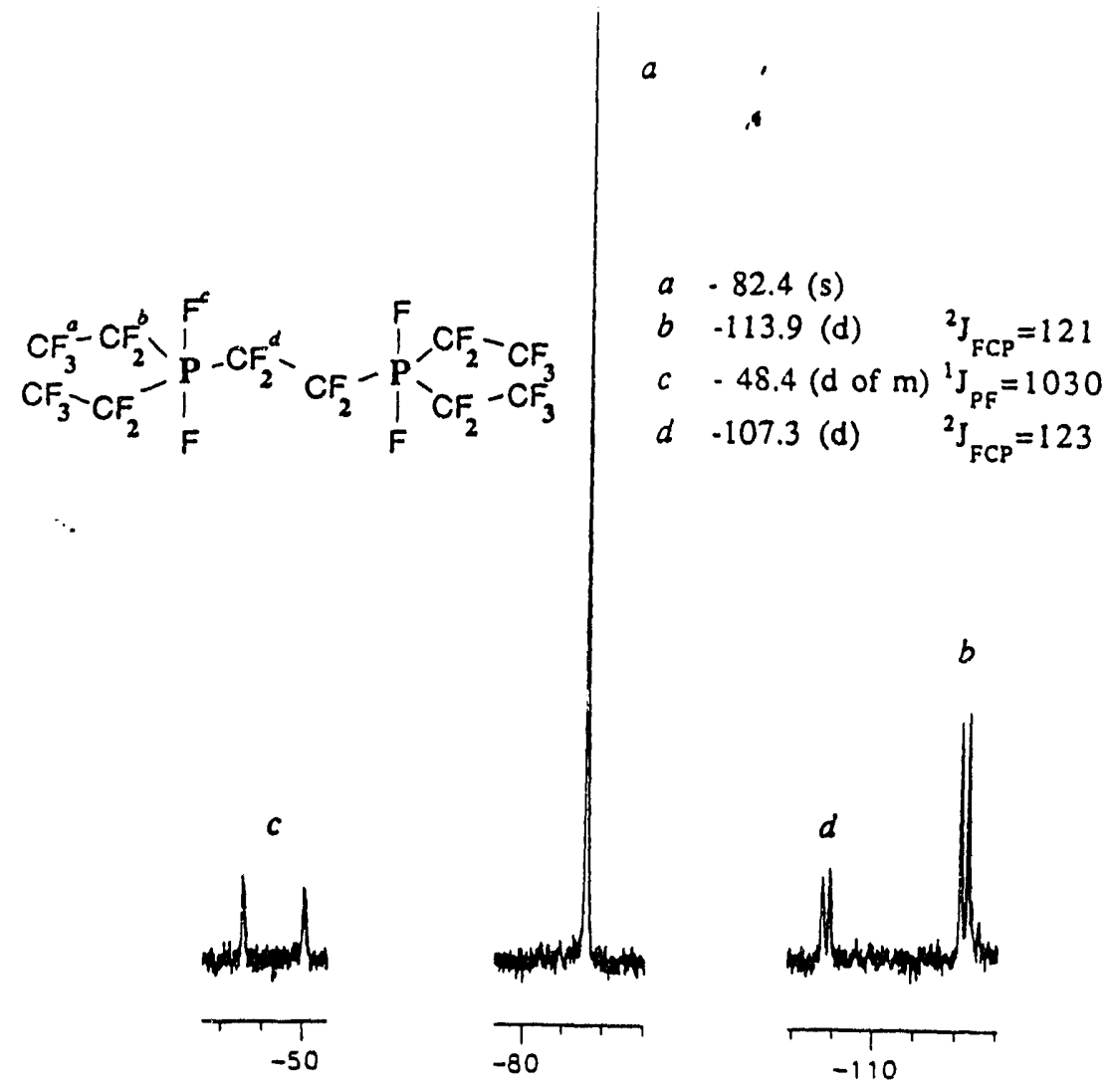

19F NMR of 1,2-bis(difluorobis(pentafluoroethyl)phosphorano)tetrafluoroethane 


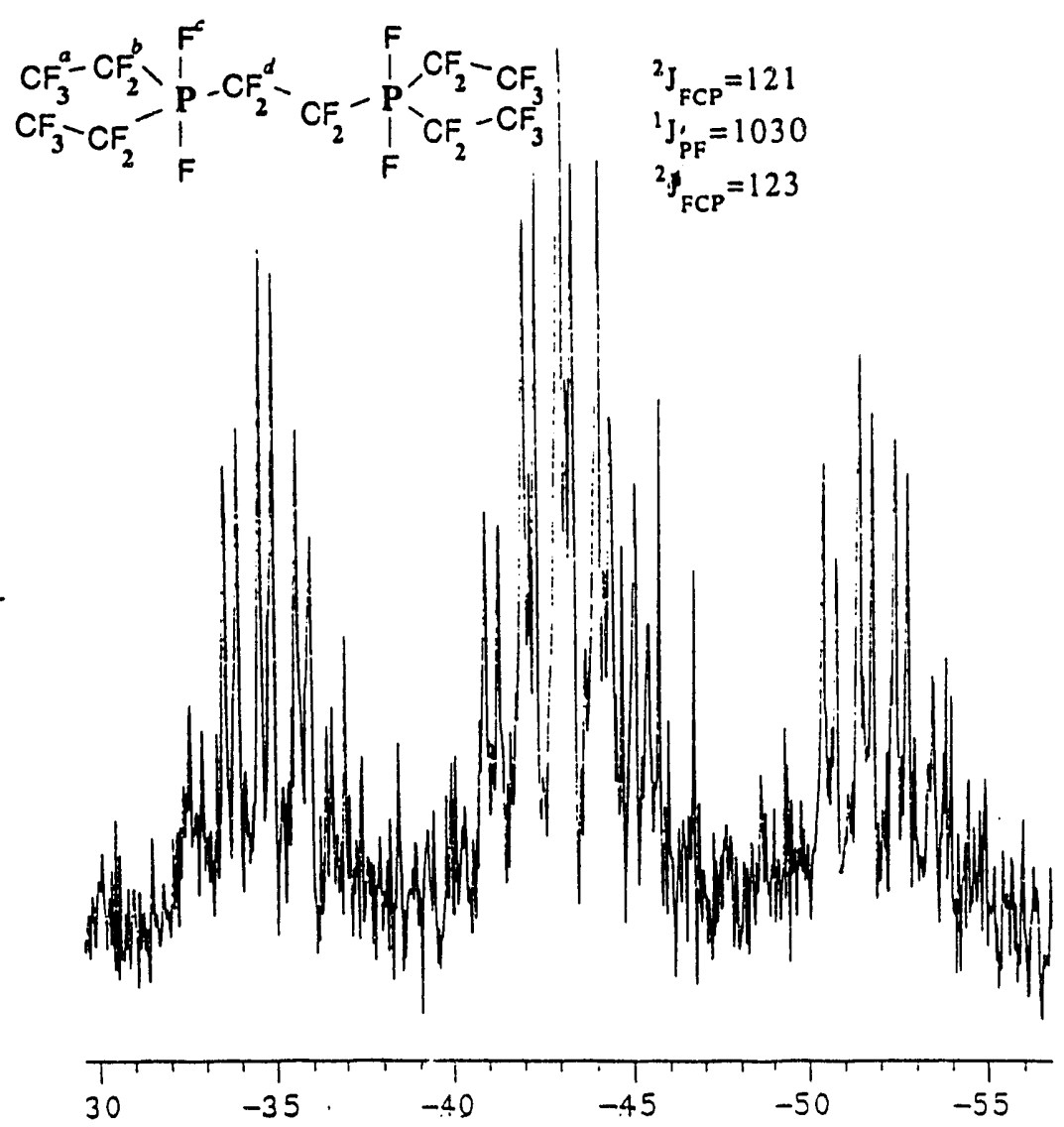

31P NMR of 1,2-bis(difluorobis(pentafluoroethyl)phosphorano)tetrafluoroethane

${ }^{19}$ E NivR Chemical Shifts Coupling Constants

$\begin{array}{ll}a-55.2 \mathrm{ppm} \text { (d of mult.) } & { }^{1} \mathrm{~J}_{\mathrm{PF}}=1068 \mathrm{~Hz} \\ b-64.7 \mathrm{ppm} \text { (d) } & { }^{2} \mathrm{~J}_{\mathrm{FCP}}=162 \mathrm{~Hz} \\ c-109.7 \text { (d) } & { }^{2} \mathrm{~J}_{\mathrm{FCP}}=122 \mathrm{~Hz}\end{array}$

d $-177.0(\mathrm{~s})$

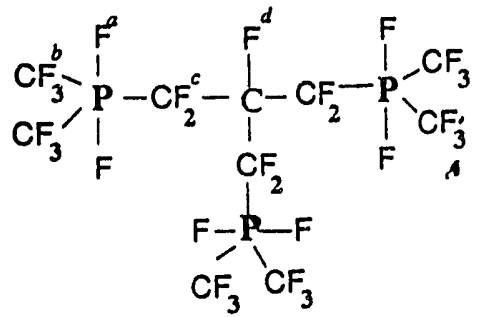

\footnotetext{
${ }^{31}$ P NMR Chemical Shift High Resolution Mass Analysis

$-49.5 \mathrm{ppm}$ ( $\mathrm{t}$ of mult.) $\mathrm{C}_{10} \mathrm{~F}_{30} \mathrm{P}_{3}{ }^{-}$Calculated: 782.873388

Observed : 782.869965
} 


\section{Preparation of perfluorinated triglyceride}

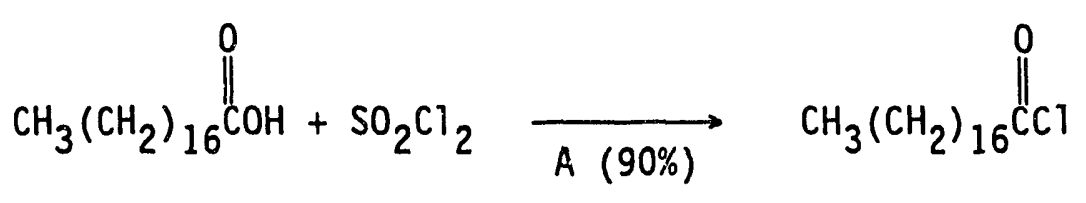

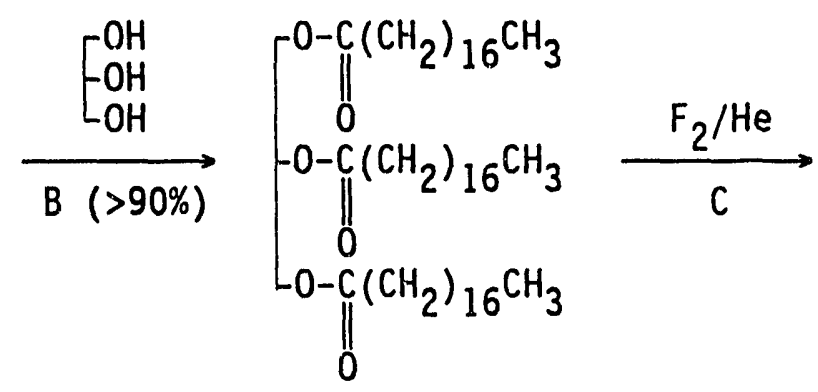

Procedure A: Thionyl chloride $(50 \mathrm{~g}, 0.42 \mathrm{~mole})$ was added to a one-neck $500 \mathrm{ml} \mathrm{flask}$. Stearic acid (100 g, $0.35 \mathrm{~mole})$ was dissolved in $50 \mathrm{ml}$ of $\mathrm{CH}_{2} \mathrm{Cl}_{2}$. The solution was added to the thionyl chloride slowly. After complete addition, the mixture was refluxed for two hours. After pumping off unreacted $\mathrm{SO}_{2} \mathrm{Cl}_{2}$, the residue was sublimated at $50{ }^{\circ} \mathrm{C}$ to get pure acid chloride in $90 \%$ yield.

Procedure B: $80 \mathrm{~g}$ of $\mathrm{CH}_{3}\left(\mathrm{CH}_{2}\right)_{16} \|_{6}^{0}$ was added slowly to a mixture of 6.7 $\mathrm{g}$ glycerin and $80 \mathrm{ml}$ pyridine at room temperature under an argon atmosphere. Two hours after addition of the acid chloride, the mixture was acidified by $10 \%$ of $\mathrm{H}_{2} \mathrm{SO}_{4}$. After filtration, the precipitate was washed with saturated $\mathrm{NaHCO}_{3}$ (aq) until it is neutral. The solid triglyceride was dried at $80{ }^{\circ} \mathrm{C}$ under high vacuum for 12 hours. The yield is higher than $90 \%$. 

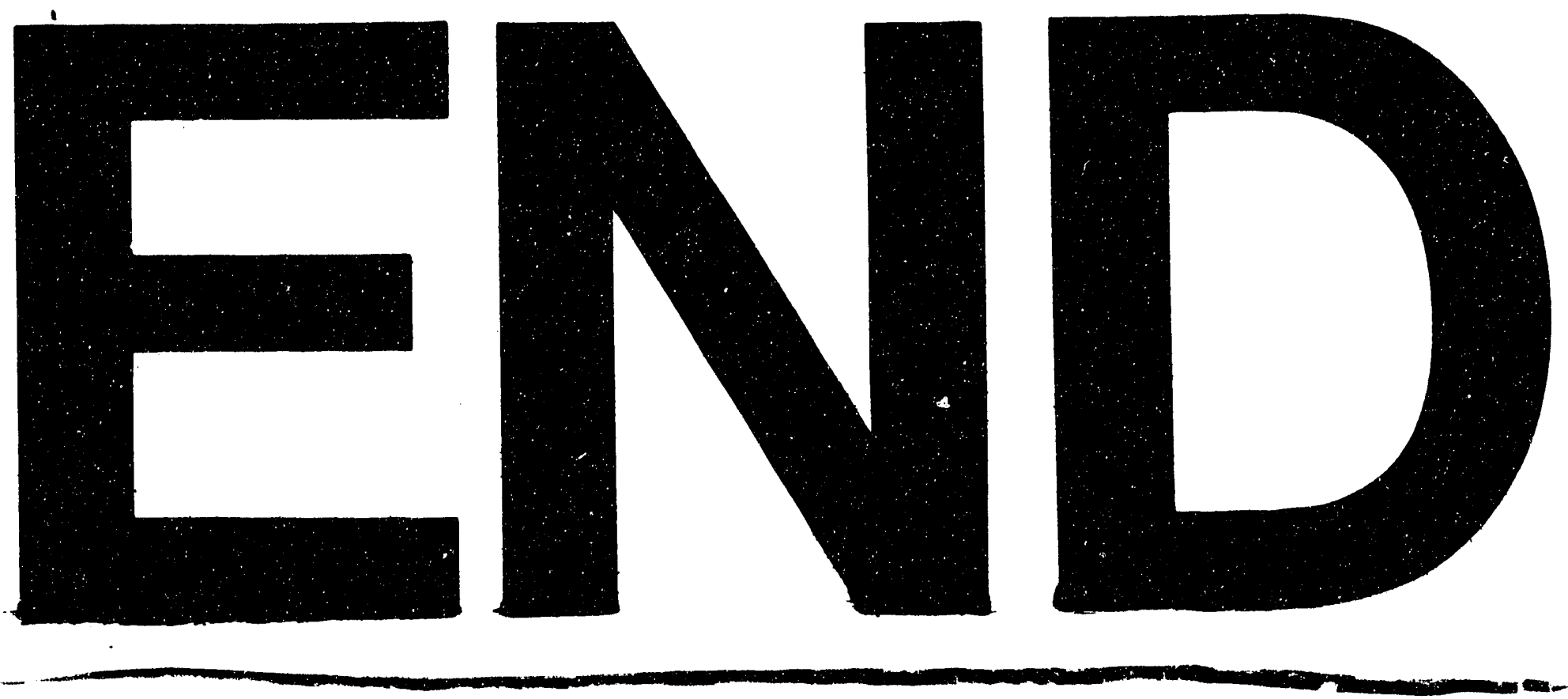
1 